\title{
Educación Ambiental y Educación Social: el punto de vista de los estudiantes
}

\author{
Francisco Javier Perales Palacios. Universidad de Granada \\ Recepción: 22 de diciembre de 2016 | Aceptado: 9 de enero de 2017 \\ Correspondencia: Francisco Javier Perales Palacios | Correo-e: fperales@ugr.es \\ iD 0000-0002-6112-2779 \\ Citar: Perales Palacios, FJ. (2017). Educación Ambiental y Educación Social: el punto de vista \\ de los estudiantes. ReiDoCrea, 6, 1-15.
}

\begin{abstract}
Resumen: Este estudio parte de un marco teórico centrado en la dimensión social de la Educación Ambiental (EA) y en el concepto de ambientalización curricular. Los objetivos son: (1) mostrar los principales elementos curriculares de una materia relacionada con la Educación Ambienta impartida en el grado de Educación Social de la Universidad de Granada; y (2) analizar los datos extraídos de diversas actividades realizadas por los alumnos tras la lectura de algunos documentos entregados previamente y que buscan aportar información relevante sobre sus percepciones y expectativas acerca de las relaciones ambiente-sociedad. Seguidamente se presenta el contexto curricular, la muestra de estudiantes y las actividades que hubieron de abordar. Posteriormente se analizan y tabulan las respuestas de los estudiantes, agrupándolas en categorías y ejemplificando, en su caso, cada una de ellas. Los resultados inciden en cuestiones como: las actitudes y comportamientos de los jóvenes en relación al medio ambiente, las posibles alternativas, los conceptos de EA y Desarrollo Sostenible (DS), necesidades vitales, conflicto entre ellas y dilemas cotidianos relacionados con el DS. Los resultados muestran una diversidad de opiniones, creencias, valoraciones y alternativas críticas ante la situación actual del Planeta que avalan la necesidad de ambientalizar curricularmente los estudios del grado de Educación Social.
\end{abstract}

Palabras clave: Educación Ambiental | Educación Social

\section{Environmental Education and Social Education: The Point of View of Students}

Abstract: This study is based on a theoretical framework centered on the social dimension of Environmental Education (EE) and the concept of curricular environmentalization is presented. The objectives are: (1) show the main curricular elements of a subject related to Environmental Education taught in the degree of Social Education the University of Granada; and (2) to analyze the data extracted from various activities carried out by the students after reading documents previously submitted and that seek to provide relevant information about their perceptions and expectations on the relationship between the environment and society. Then the curricular context, the student sample and the activities to be addressed are presented. Subsequently, the students' responses are analyzed and tabulated, grouping them into categories and exemplifying, if necessary, each of them. The results affect issues such as: the attitudes and behaviors of young people in relation to the environment, the possible alternatives, the concepts of EE and Sustainable Development (SD), vital needs, conflict among them and everyday dilemmas related to SD. The results show a diversity of opinions, beliefs, evaluations and alternatives that are highly varied and critical to the current situation of the Planet, which confirm the need to environmentalize the curriculum for the degree of Social Education.

Keywords: Environmental Education | Social Education

\section{Introducción}

El concepto de Educación Ambiental, a pesar de sus orígenes relativamente recientes, ha ido modificando gradualmente su significado y ámbito de aplicación. Esta evolución fue sintetizada inicialmente por Lucas (1972) mediante tres preposiciones: "en, sobre y para el ambiente". Con ellas dicho autor quiso evidenciar la transición desde una EA centrada en la inmersión en el medio natural hasta una dimensión más ligada a su defensa activa. 
Por otra parte, la definición de la Unión Internacional de Conservación de la Naturaleza (UICN) en el año 1970 ya reflejaba la dimensión cultural entre las competencias de la EA: Proceso de reconocer valores y clarificar conceptos, en el orden de desarrollar las destrezas y actitudes necesarias para comprender y apreciar las interrelaciones entre el hombre, su cultura y su entorno biofísico. La Educación Ambiental también implica la práctica en la toma de decisiones y en la autoformación de un código de conducta acerca de las cuestiones que afectan a la calidad ambiental (Carta de Nevada, UICN, 1970; citada en Alonso, 2010).

Con las dos referencias anteriores queremos poner de manifiesto que el concepto de EA ha ido transformándose en un doble sentido, por un lado, desde un naturalismo pasivo a un activismo con un marcado acento ecologista y, por el otro, desde una consideración del medio natural como objeto exclusivo de atención hasta la toma de conciencia de su interconexión con la dimensión social (cultura, economía, filosofía...). En paralelo a este camino recorrido, han surgido otras denominaciones para la EA que han pretendido sustituirla o enriquecerla, tal es el caso de la Educación para el Desarrollo Sostenible (EDS) o para un Futuro Sostenible y que podrían integrarse en una nueva disciplina conocida como Ciencia de la Sostenibilidad (Vilches y Gil, 2015).

Al margen de estas disquisiciones semánticas, deseamos fijar nuestra atención en la necesidad de vincular los objetivos de la EA a la Sociedad en la que se halla inmersa con el fin de intentar hacerla cómplice en la consecución de los objetivos señalados y, de esta forma, asegurar una extensión y continuidad de aquellos.

En este trabajo vamos a centrarnos en describir una experiencia llevada a cabo con estudiantes del grado de Educación Social de la Universidad de Granada a través de una asignatura optativa incluida en su plan de estudios y cuya denominación es: Ámbitos, contextos e instrumentos de la Educación Ambiental.

Como corresponde a las intenciones de este trabajo, vamos a abundar en dos fuentes teóricas principalmente: (1) las que proceden propiamente de la dimensión social de la EA y (2) las que se corresponden con la ambientalización del currículo (también denominada sostenibilidad curricular).

\section{La dimensión social de la EA}

Actualizando la definición de la UICN, podemos decir que la EA es concebida como el proceso individual y colectivo mediante el cual los individuos y su comunidad toman conciencia de los problemas ambientales, y adquieren actitudes, aptitudes y valores que los llevan a actuar para intentar resolverlos. Desde esta óptica resolutiva (Sauvé, 2004) se aborda el objeto de estudio como un todo complejo que básicamente se analiza como un sistema de relaciones, con una perspectiva dialógica (pensamientosentimiento, diálogo entre disciplinas) y hologramática (diferencias y complementación de escalas micro-meso-macro).

A este respecto, lo social se advierte en la dimensión colectiva precisa para la resolución de los problemas ambientales, en la necesaria cooperación para su abordaje y en la propia naturaleza de los problemas ambientales, al punto de ser denominados frecuentemente como problemas socioambientales. Un problema clásico de actualidad, como es el cambio climático, afecta no solo a los ecosistemas (deshielo, subida del nivel del mar consecuente, mayor frecuencia de desastres naturales...) sino a las sociedades más vulnerables (poblaciones costeras, asentamientos frágiles, agricultura de subsistencia...) y su resolución demanda acuerdos internacionales por parte de gobiernos pero que implican a empresas, asociaciones y otros agrupamientos 
sociales en un deseable cambio de hábitos colectivo (en este caso relacionado especialmente con el abandono de fuentes de energía fósiles).

En su versión de la EA como EDS, una revisión de expertos estima que los esquemas y procesos de aprendizaje incluyen las siguientes características clave:

- Procesos de colaboración y diálogo (incluyendo a los tomadores de decisiones y el diálogo intercultural).

- Procesos que incluyen el 'sistema global'.

- Procesos con currículo y experiencias de enseñanza y aprendizaje innovadoras.

- Y procesos de aprendizaje activo y participativo (Tilbury, 2011, p. 7).

Como podemos comprobar, la dimensión social se halla presente, al menos, en el primer y cuarto grupo de procesos.

Igualmente, Tilbury (2011, p. 9) considera, mediante una revisión de estudios de caso, que los procesos de aprendizaje también pueden contribuir en un amplio rango al cambio económico, ambiental, social (incluyendo lo cultural) y educativo.

La perspectiva psicoeducativa de esta visión social de los problemas ambientales hunde sus raíces en el Constructivismo Socio-cultural que contempla el aprendizaje como una interacción activa entre las concepciones previas de los individuos y el nuevo conocimiento compartido socialmente, y cuyos principales artífices han sido Piaget, Bruner, Vygotsky y von Glasersfeld (Stauffacher, Walter, Lang, Wiek y Scholz, 2006). Desde esta visión, el conocimiento no se contempla como algo objetivo e individual sino como un producto colectivo que se comparte socialmente.

Desde una perspectiva más próxima a las intenciones de este estudio, otros autores han encontrado un profundo paralelismo entre la EA y la Educación Social (ES, en adelante). Así, Iglesias y Meira (2007) encuentran profundas semejanzas entre ambas desde su propio surgimiento hasta su status actual. En palabras de estos autores:

Cualquier problema ambiental convertido en objeto educativo, sea cual sea el ámbito espacial en el que se desarrolle la acción (local, regional, global), obliga a una práctica de contextualización y problematización que es, en esencia, una práctica de acción social o socioeducativa (Iglesias y Meira, 2007, p. 15).

En la misma línea, De la Osa y Azara (2012) afirman que la EA es considerada por la ES como uno de sus posibles ámbitos de desarrollo y con trayectorias convergentes, proponiendo como ejemplo de esa convergencia a la "Estrategia Aragonesa de Educación Ambiental". Igualmente, Amador y Esteban (2011) encuentran coincidencias en los fines de la EA y la ES, reclamando el surgimiento de una línea de investigación denominada intervención educativa socioambiental.

Seguramente las principales dificultades para lograr una mayor y mejor aproximación entre la EA y la ES sea la diferente formación universitaria recibida por los educadores ambientales y los educadores sociales. De hecho, al margen de la asignatura que nos compete, existen escasos precedentes curriculares sobre presencia de materias de EA en los estudios universitarios de ES, en concreto uno de ellos lo oferta la UNED. Por el contrario, sí existe una presencia significativa de la EA en los planes de estudio de Ciencias Ambientales en España (Luque y Perales, 2016). 


\section{La ambientalización curricular}

La EA ha tenido tradicionalmente cierta cabida en la educación obligatoria desde diferentes posiciones (García, 2004):

- Como conocimiento ecológico del medio, pero también en la consideración del propio contexto educativo como nicho ecológico.

- Como activismo en la defensa y protección del medio ambiente (p. ej., el "día de la bicicleta").

- Como reformulación del propio conocimiento escolar en aspectos tales como: la consideración de lo sociocultural dentro del concepto de medio, la incorporación del componente axiológico del conocimiento (actitudes, valores, normas, creencias) o como núcleo de integración de los contenidos escolares (p. ej., a partir de ideas-eje como el agua o la energía, de manera que las disciplinas se ponen a su servicio).

Dicha incorporación no se halla exenta de dificultades, tales como:

- La imposibilidad de considerar la EA como una asignatura del currículo, dado su carácter global.

- La necesidad de que afecte a todas las materias escolares, lo que exige una formación y concienciación del profesorado y una coordinación del mismo, así como compatibilizar la naturaleza poco convencional de la EA con la más tradicional propia de las disciplinas clásicas del currículo.

- La demanda de métodos activos de enseñanza y aprendizaje para no hurtar a los alumnos los grandes fines de la EA.

Podemos considerar la ambientalización curricular como la impregnación de la dimensión ambiental en los currículos prescritos oficialmente. En el caso español, los dilemas sobre cómo articular la EA en el marco de la Ley General del Sistema Educativo Español (LOGSE), se resolvió a favor de la invención de las llamadas "áreas transversales" que, como su denominación indica, "atravesarían" las áreas curriculares clásicas (Lengua, Ciencias de la Naturaleza, Matemáticas...). Aparte de la propia EA, algunas otras temáticas transversales han sido: salud, consumo, sexualidad, desarrollo humano, etc. Estos temas han aportado, al margen de la tradicional focalización en el conocimiento conceptual, un mayor interés por los valores, los compromisos éticos y por un posicionamiento crítico ante la realidad. No obstante y, como apuntábamos más arriba al referirnos a las dificultades de la ambientalización del currículum, este planteamiento es contradictorio, quedándose a medio camino entre una integración plena de la EA y la incorporación como conjunto de actividades extraescolares o puntuales.

La ambientalización del currículo también alberga riesgos, uno de ellos puede ser la desnaturalización de lo ambiental. Por ejemplo, Feinstein y Kirchgasler (2015) señalan que la inclusión de lo ambiental en los estándares de ciencia en los Estados Unidos puede inducir "la posibilidad de una idea sobre-simplificada de la Sostenibilidad que disminuya sus dimensiones éticas y sociales exagerando el papel de la tecnología..." (Feinstein y Kirchgasler (2015, p. 123).

En el caso de la Universidad, en cuanto nicho de formación de las futuras clases dirigentes, constituye un contexto idóneo para la ambientalización del currículo; no obstante se carece, en general, de una voluntad decidida para ello y de unos medios técnicos y humanos acordes a los objetivos de la EA. En cualquier caso las iniciativas que se adopten deberían conllevar rigurosos estudios sobre su viabilidad y las 
competencias adquiridas (Hansmann, 2009) a modo de evaluación mediante indicadores consensuados como los planteados por Medir, Heras y Magin (2016).

Aramburuzabala, Cerrillo y Tello (2015) hacen hincapié en las estrategias didácticas que deberían acompañar a la ambientalización curricular universitaria, tales como: Las dialógicas (discusión, debate, diálogo); la reflexión crítica sobre temas relevantes a nivel local y global; casos hipotéticos y reales (role play y estudio de casos); colaboración en proyectos y tareas (actividades en grupo); actividades de aprendizaje experiencial como la acción comunitaria y la resolución de problemas; mentorías (para facilitar la comprensión y el compromiso con los temas de la EDS); exploración y resolución de problemas interdisciplinares; aprendizaje en la acción (en el que los aprendices desarrollan e implementan planes de acción, reflexionan sobre su experiencia y sugieren mejoras); y la investigación-acción (proceso cíclico de planificación, acción, observación y reflexión basado en la investigación para innovar y mejorar la práctica) (Aramburuzabala y otros, 2015, pp. 84-85).

A estos efectos, dichos autores defienden el "aprendizaje-servicio" como la alternativa que mejor sintetiza los principios anteriores.

En España han tenido lugar algunas iniciativas donde puede enmarcarse la ambientalización curricular, como ha sido la creación de la CADEP (Comisión sectorial de Calidad Ambiental, Desarrollo Sostenible y Prevención de Riesgos), perteneciente a la CRUE (Conferencia de Rectores de las Universidades Españolas), y la Red ACES de Ambientalización de los Estudios Superiores. En concreto la CADEP, en su documento "Orientaciones para la introducción de la sostenibilidad en el curriculum", propone "formar profesionales comprometidos con la sostenibilidad, teniendo en cuenta como criterios orientadores seis principios en el proceso educativo universitario: el principio ético, el holístico, el de la complejidad, el de globalización, el de transversalidad y el de responsabilidad social universitaria" (CADEP-CRUE, 2005, p. 8). Asimismo, la Red ACES define Sostenibilidad Curricular como un proceso continuo de producción cultural para formar ciudadanos, profesionales comprometidos con la búsqueda permanente de las mejores relaciones posibles sociedad-naturaleza, atendiendo a los valores de justicia, solidaridad, equidad y el respeto a las diversidades (Geli, 2002, citada en Jiménez-Fontana, García, Azcárate y Navarrete, 2015).

Como vemos, nuevamente se pone de manifiesto el importante papel que han de jugar las relaciones naturaleza-sociedad. Este hecho, junto con la singularidad del colectivo participante en esta investigación -futuros educadores sociales-, estimamos que justifica la necesidad de la misma.

\section{Objetivos}

Como anunciamos previamente, en este trabajo pretendemos describir la experiencia curricular desarrollada con la materia de Ámbitos, contextos e instrumentos de la EA constituyente del grado de ES, lo que se concreta básicamente en:

1. Mostrar los principales elementos curriculares de la materia referida.

2. Analizar los datos extraídos de diversas actividades realizadas por los alumnos que, a nuestro juicio, aportan información relevante sobre sus percepciones y expectativas acerca de las relaciones ambiente-sociedad. 


\section{Métodos}

\section{El contexto curricular}

El grado de ES se genera en España en el marco de la reforma educativa universitaria vinculada al Espacio Europeo de Educación Superior. En el plan de estudios de la Universidad de Granada se incluyó una asignatura optativa para el segundo cuatrimestre del tercer curso, con seis créditos, denominada Ámbitos, contextos e instrumentos de la Educación Ambiental ${ }^{2}$. Dicha asignatura ya disponía del precedente de otra materia en la extinta Diplomatura de ES. Se trata de una materia compartida por dos áreas de conocimiento: Didáctica de las Ciencias Experimentales y Métodos de Investigación y Diagnóstico en Educación, con una tradición de cooperación a nivel docente e investigador (Perales y Gutiérrez, 2010).

Destaquemos sus objetivos:

1. Adquirir conocimientos socio-ambientales y socio-tecnológicos.

2. Comprometerse éticamente con los problemas sociales y la sostenibilidad global y ambiental.

3. Valorar la importancia de la EA para el DS en contextos no formales e informales.

4. Desarrollar competencias relacionadas con el saber hacer de programas e intervenciones de EA en ámbitos no formales e informales (estrategias, herramientas, planificación, aplicación, evaluación).

5. Desarrollar habilidades de comunicación interpersonal en gestión y desarrollo de proyectos socio-ambientales.

Su programa se estructura básicamente en dos partes, asumidas respectivamente por sendos profesores de las áreas de conocimiento señaladas anteriormente. Dichas partes contemplan los siguientes contenidos:

1. Introducción a la EA y a la Sostenibilidad. Problemáticas ambientales actuales. Estrategias metodológicas en la EA. Intervención en la Educación formal.

2. TIC y alfabetizacion ambiental. Ecologismo y Movimientos proambientales. Gestión e Intervención educativa en contextos no formales e informales. Evaluación de programas, centros, materiales, recursos y cambios personales. Competencias profesionales y perfil del educador social en programas de EA.

La metodología de enseñanza combina distintas estrategias recomendadas para la EA (Aramburuzabala y otros, 2015), tales como las dialógicas, reflexión crítica, juego de roles, aprendizaje cooperativo, resolución de problemas ambientales y trabajo por proyectos. Ello desemboca en actividades presenciales y no presenciales en torno a las siguientes temáticas:

- Profundización en problemáticas ambientales actuales.

- Análisis de medios de comunicación sobre noticias de contenido ambiental e interés social.

- Salidas y visitas en el entorno.

- Diseño, aplicación y evaluación de proyectos de EA en el ámbito de actuación no formal e informal. 


\section{Muestra participante}

Los resultados que se muestran corresponden al curso 2015-16, en el que se matricularon 66 estudiantes, de los que 16 eran des sexo masculino y 50 del femenino.

\section{Actividades e instrumentos de análisis}

Vamos a ir describiendo cada una de las actividades propuestas al alumnado objeto de análisis en este artículo.

\section{Actividad 1}

Dicha actividad se presenta como introducción a la asignatura. En ella se muestra una noticia aparecida en el canal de comunicación de la Universidad de Granada el día 83-2011 y titulada "Aprobados en teoría, suspensos en práctica", donde se describen los resultados de una encuesta realizada entre estudiantes de la Universidad de Granada con ítems similares a los utilizados en los eurobarómetros. La noticia comenta las respuestas sobre comportamientos relativos a residuos, ahorro de agua y energía, movilidad, contaminación acústica, consumo de productos ecológicos y turismo sostenible. Asimismo se pregunta sobre su participación en iniciativas locales de índole medioambiental o en asociaciones ecologistas y, en caso negativo, si les gustaría hacerlo. Tal noticia fue elegida tanto por referirse a estudiantes próximos geográficamente a los de la muestra participante como por resaltar en su título una de las claves para entender la insuficiente implicación de los jóvenes con los comportamientos proambientales.

Tras su lectura en clase, se pide a los alumnos que de forma individual respondan a las siguientes cuestiones:

1. ¿Qué reflexión os merece la lectura anterior?

2. ¿Os sentís identificados con los datos que se ofrecen?

3. ¿Qué podría hacerse para cambiar esta situación?

Las respuestas fueron sometidas a un análisis de contenido en el que, de modo inductivo, se identificaron unidades de significado, se agruparon por similitud y se generaron categorías.

\section{Actividad 2}

Tras una introducción a la EA, su evolución histórica y principales conceptos y procedimientos relevantes (problemas ambientales, huella ecológica, dimensión social y científica...), esta actividad se introduce para hacer reflexionar a los estudiantes sobre el concepto de DS (cuestión a, Anexo I). La lectura y cumplimentación de la misma pudieron realizarla los alumnos fuera del horario de clase. Consta de los siguientes apartados:

1. Se les suministran varios documentos escritos para que los lean: (a) "El guano: un caso de agotamiento de los recursos" (Tudela, 1990, p. 78); (b) "Los límites del crecimiento" (Meadows, Meadows, Randers y Behrens, 1972, p. 23); (c) Los problemas del Producto Interior Bruto (Ekins, 1986, pp. 8-9, 38, 128-129, 133); y (d) DS (Conroy y Litvinoff, 1988, p. XI).

2. Se les solicita a continuación: "Analizar el concepto de Desarrollo Sostenible y llevarlo a nuestras vidas". Para ello se les suministra una definición clásica del DS: "Es el desarrollo que satisface las necesidades actuales de las personas 
sin comprometer la capacidad de las futuras generaciones para satisfacer las suyas" (Comisión Brundtland, 1987). A partir de ahí se les formulan seis interrogantes consecutivos que mostraremos posteriormente.

El análisis de las respuestas se realizó de modo similar al de la Actividad 1, organizándose a partir de las cuestiones que les fueron formuladas.

\section{Resultados}

Vamos a ir mostrándolos para cada actividad de aula.

\section{Actividad 1}

La actividad la realizaron un total de 50 estudiantes. Iremos describiendo las categorías generadas en el análisis de contenido realizado para cada una de las cuestiones que conforman esta actividad. Para ello tanto en esta actividad como en la 2 solo representaremos las respuestas con porcentajes mayores o iguales a un 5 por ciento.

\section{1. ¿Qué reflexión os merece la lectura anterior?}

En esta pregunta se produjeron un total de 84 respuestas distintas, lo que hace un total de 1,68 respuestas por individuo. El término más frecuente fue el de "concienciación" ( $\mathrm{N}=32)$, que fue citado como existente por 9 , no existente por 14 , necesario por 8 y otra respuesta no clasificable. En la Tabla I presentamos los resultados, mostrando la denominación de la categoría, la frecuencia relativa de respuestas y un ejemplo de las mismas.

\begin{tabular}{|c|c|c|}
\hline $\begin{array}{l}\text { CATEGORÍAS DE } \\
\text { RESPUESTA }\end{array}$ & $\begin{array}{l}\text { PORCENTAJE DE } \\
\text { RESPUESTAS }\end{array}$ & EJEMPLO DE RESPUESTAS \\
\hline $\begin{array}{l}\text { Ratificación de los resultados } \\
\text { globales de la encuesta }\end{array}$ & $35 \%$ & $\begin{array}{l}\text { Hay mucha información pero la gente no tiene } \\
\text { compromiso con lo que es cuidar el medioambiente }\end{array}$ \\
\hline $\begin{array}{l}\text { Causas }(\mathrm{N}>1) \text { : } \\
\text { - Falta de conciencia/actitud } \\
\text { - Por condicionamiento } \\
\text { económico } \\
\text { - No se facilita } \\
\text { institucionalmente } \\
\text { - Por condicionamiento social }\end{array}$ & $29 \%$ & Interesa más lo económico que el medioambiente \\
\hline $\begin{array}{l}\text { Escepticismo ante los } \\
\text { resultados de la encuesta }\end{array}$ & $11 \%$ & $\begin{array}{l}\text { La actitud de los jóvenes a que se refiere la encuesta no } \\
\text { coincide con lo que observo a mi alrededor }\end{array}$ \\
\hline Soluciones de tipo formativo & $8 \%$ & $\begin{array}{l}\text { Aunque hay mucha gente que cree que está } \\
\text { concienciada, se desconocen algunas acciones que } \\
\text { perjudican al medioambiente }\end{array}$ \\
\hline Sin clasificar & $17 \%$ & $\begin{array}{l}\text { Se derrocha mucho dinero en campañas de } \\
\text { concienciación pero gran parte de la gente no se lo toma } \\
\text { en serio }\end{array}$ \\
\hline
\end{tabular}

\section{2. ¿Os sentís identificados con los datos que se ofrecen?}

Esta cuestión generó un total de 92 respuestas con distinto significado, lo que representa un promedio de respuestas por individuo de 1,84, es decir, mayor que en la cuestión primera. Globalmente las respuestas tuvieron un sentido afirmativo (explícito 
o implícito) en 69 ocasiones, negativo en 5 y parcialmente en 11. En la Tabla II se hace una representación similar a la de la Cuestión 1.

\begin{tabular}{|c|c|c|}
\hline $\begin{array}{l}\text { CATEGORIAS DE } \\
\text { RESPUESTA }\end{array}$ & $\begin{array}{l}\text { PORCENTAJE DE } \\
\text { RESPUESTAS }\end{array}$ & EJEMPLO DE RESPUESTAS \\
\hline $\begin{array}{l}\text { Reciclo/ahorro agua/no arrojo residuos al } \\
\text { suelo }\end{array}$ & $21 \%$ & Intento reciclar o ahorrar agua \\
\hline $\begin{array}{l}\text { Causas }(\mathrm{N}>1) \text { : } \\
\text { - Por desconocimiento/se precisa más } \\
\text { información } \\
\text { - Se ahorra agua por economía } \\
\text { - Por bienestar o individualismo } \\
\text { - No somos verdaderamente conscientes } \\
\text { de su importancia }\end{array}$ & $18 \%$ & $\begin{array}{l}\text { Nos preocupa más nuestro bienestar que el } \\
\text { entorno y actuamos individualmente }\end{array}$ \\
\hline $\begin{array}{l}\text { Diferencia entre actitud/ conocimiento y } \\
\text { conciencia/ comportamiento }\end{array}$ & $13 \%$ & $\begin{array}{l}\text { Hay conocimiento teórico de cómo se debe } \\
\text { actuar pero por motivos diversos no se hace }\end{array}$ \\
\hline $\begin{array}{l}\text { Preferencia por productos ecológicos } \\
\text { pero hay impedimentos económicos }\end{array}$ & $11 \%$ & $\begin{array}{l}\text { Me gustaría comprar productos ecológicos } \\
\text { pero por mi situación económica no puedo }\end{array}$ \\
\hline Podría hacer o aprender más & $9 \%$ & $\begin{array}{l}\text { Tengo algo de conciencia pero no hago todo lo } \\
\text { que debería hacer }\end{array}$ \\
\hline Ejemplos de situaciones concretas & $9 \%$ & $\begin{array}{l}\text { En mi casa nunca hemos reciclado pero he de } \\
\text { dar el paso y concienciar a mi familia }\end{array}$ \\
\hline
\end{tabular}

\section{3. ¿Qué podría hacerse para cambiar esta situación?}

Para esta cuestión se generaron en total 121 respuestas, lo que representa un promedio de 2,42 respuestas por individuo, claramente superior a los de las cuestiones anteriores, lo que manifiesta un importante grado de iniciativa. En la Tabla III se representan los datos de forma análoga a lo realizado en las cuestiones 1 y 2 .

\begin{tabular}{|c|c|c|}
\hline \multicolumn{3}{|c|}{$\begin{array}{l}\text { Tabla III. Categorías identificadas en las respuestas }(N=121) \text { de los estudiantes }(N=50) \text { a la Cuestión } 3 \text { de la } \\
\text { Actividad 1, porcentaje de las mismas frente al total y ejemplos de las respuestas categorizadas }\end{array}$} \\
\hline $\begin{array}{l}\text { CATEGORÍAS DE } \\
\text { RESPUESTA }\end{array}$ & $\begin{array}{l}\text { PORCENTAJE DE } \\
\text { RESPUESTAS }\end{array}$ & EJEMPLO DE RESPUESTAS \\
\hline $\begin{array}{l}\text { Educación (EA/formación/ } \\
\text { información/sensibilización) ( } \mathrm{N}>1) \text { : } \\
\text { - Desde edades tempranas en los colegios } \\
\text { - En los hogares } \\
\text { - Sobre los perjuicios en el medioambiente y } \\
\text { las consecuencias } \\
\text { - Mediante campañas para hacer consciente } \\
\text { a la población y que actúe } \\
\text { - Ambientalizando el currículo }\end{array}$ & $44 \%$ & $\begin{array}{l}\text { Fomentar desde Infantil el respeto al } \\
\text { medioambiente y hacerlo también en la casa }\end{array}$ \\
\hline $\begin{array}{l}\text { Promoviendo una conciencia } \\
\text { activa/compromiso }\end{array}$ & $10 \%$ & $\begin{array}{l}\text { Mayor concienciación ciudadana y } \\
\text { compromiso }\end{array}$ \\
\hline $\begin{array}{l}\text { Mediante normativas públicas con incentivos } \\
\text { o sanciones }\end{array}$ & $8 \%$ & Establecer cupos de consumo de agua \\
\hline $\begin{array}{l}\text { Mejorando la oferta/precio de productos } \\
\text { ecológicos }\end{array}$ & $7 \%$ & Mediante ayudas para bajar su precio \\
\hline Incidiendo en la práctica & $7 \%$ & $\begin{array}{l}\text { Centrándonos más en la práctica pero sin } \\
\text { olvidar la teoría }\end{array}$ \\
\hline Sin clasificar & $10 \%$ & Cambiar de estilo de vida \\
\hline
\end{tabular}




\section{Actividad 2}

La actividad en cuestión fue respondida por 58 estudiantes. Seguidamente expondremos los resultados del análisis de sus respuestas.

\section{4. ¿Qué relación existe entre EA y DS?}

En esta primera reflexión se distinguieron 74 respuestas diferentes, por lo que se obtuvo un índice 1,28 respuestas por individuo, bastante inferior a los obtenidos en la Actividad 1, lo que podría ser explicado porque los estudiantes centran más sus respuestas a partir de lo ya estudiado en la asignatura. La Tabla IV muestra los resultados de manera análoga a las tablas anteriores.

\begin{tabular}{|c|c|c|}
\hline $\begin{array}{l}\text { CATEGORÍAS DE } \\
\text { RESPUESTA }\end{array}$ & $\begin{array}{l}\text { PORCENTAJE } \\
\text { DE RESPUESTAS }\end{array}$ & EJEMPLO DE RESPUESTAS \\
\hline $\begin{array}{l}\text { La EA es un proceso/ } \\
\text { instrumento/herramienta/medio para } \\
\text { alcanzar/favorecer/su finalidad es el } \\
\text { DS }\end{array}$ & $34 \%$ & $\begin{array}{l}\text { La EA es un proceso fundamental cuyo objetivo } \\
\text { principal es la consecución del DS, el cual se } \\
\text { encarga de mantener y satisfacer las necesidades } \\
\text { de la comunidad asegurando su calidad de vida, sin } \\
\text { que los diversos factores por los que están } \\
\text { influenciados, repercutan negativamente en su } \\
\text { desarrollo. }\end{array}$ \\
\hline $\begin{array}{l}\text { El DS precisa de la EA como } \\
\text { herramienta para el cambio social }\end{array}$ & $18 \%$ & $\begin{array}{l}\text { Es obvio que no podemos hablar de DS sin } \\
\text { mencionar la EA como herramienta de cambio y de } \\
\text { concienciación de todos los agentes políticos } \\
\text { sociales, provocando asi una sociedad estable y que } \\
\text { consuma solo lo necesario, siendo conscientes de } \\
\text { que estamos destruyendo lo que nos rodea por } \\
\text { encima de nuestras posibilidades. }\end{array}$ \\
\hline $\begin{array}{l}\text { Son términos correlativos/ } \\
\text { complementarios/están muy } \\
\text { relacionados }\end{array}$ & $18 \%$ & $\begin{array}{l}\text { En relación con la EA y el DS puedo decir que los } \\
\text { dos conceptos están muy ligados entre ellos, ya que } \\
\text { ambos buscan que las personas puedan vivir de } \\
\text { manera saludable sin la necesidad de explotar los } \\
\text { recursos naturales. }\end{array}$ \\
\hline $\begin{array}{l}\text { Se hace una crítica al consumismo y a } \\
\text { la sociedad capitalista }\end{array}$ & $8 \%$ & $\begin{array}{l}\text { Considero que la base errónea de este sistema } \\
\text { educativo reside en una implantación de un sistema } \\
\text { capitalista que premia al que más tiene, y no hace lo } \\
\text { mismo con el que más cuida de su medio.. }\end{array}$ \\
\hline $\begin{array}{l}\text { El DS está más orientado a la acción y } \\
\text { la EA enseña a cómo hacerlo }\end{array}$ & $5 \%$ & $\begin{array}{l}\text { El DS promueve acciones de cuidado del } \\
\text { medioambiente y la EA enseña a cómo cuidarlo }\end{array}$ \\
\hline El DS orienta la estrategia de la EA & $5 \%$ & $\begin{array}{l}\text { EI DS es una de las principales acciones que debe } \\
\text { transmitir la EA }\end{array}$ \\
\hline Sin clasificar & $12 \%$ & DS: parte muy importante incluida en la EA. \\
\hline
\end{tabular}

\section{Tras la lectura de los siguiente documentos debes dar un concepto de personal acerca del DS}

La segunda cuestión planteada a los estudiantes generó $\mathrm{N}=72$ respuestas diferentes, o lo que es lo mismo, 1,24 respuestas por individuo, es decir, índice muy similar al de la cuestión (a). En la Tabla $\mathrm{V}$ exponemos los resultados de forma análoga a como lo hemos hecho en las tablas anteriores. 


\begin{tabular}{|c|c|c|}
\hline \multicolumn{3}{|c|}{$\begin{array}{l}\text { Tabla V. Categorías identificadas en las respuestas }(\mathrm{N}=72) \text { de los estudiantes }(\mathrm{N}=58) \text { a la Cuestión (b) de la } \\
\text { Actividad 2, porcentaje de las mismas frente al total y ejemplos de las respuestas categorizadas }\end{array}$} \\
\hline $\begin{array}{l}\text { CATEGORIAS DE } \\
\text { RESPUESTA }\end{array}$ & $\begin{array}{l}\text { PORCENTAJE } \\
\text { DE RESPUESTAS }\end{array}$ & EJEMPLO DE RESPUESTAS \\
\hline Definición clásica & $26 \%$ & Véase nota a pie de página $n^{\circ} 2$. \\
\hline $\begin{array}{l}\text { Cubrir necesidades esenciales } \\
\text { protegiendo al medio }\end{array}$ & $18 \%$ & $\begin{array}{l}\text { El DS, desde mi punto de vista, consiste en cubrir } \\
\text { las necesidades de todas las personas respetando al } \\
\text { medio y sin sobreexplotarlo. }\end{array}$ \\
\hline Mantener los recursos del planeta & $10 \%$ & $\begin{array}{l}\text { Mantener los recursos del planeta para que no se } \\
\text { agoten y se mantengan para las generaciones } \\
\text { futuras. }\end{array}$ \\
\hline $\begin{array}{l}\text { Crítica al sistema capitalista y a la } \\
\text { sobreexplotación de recursos }\end{array}$ & $7 \%$ & $\begin{array}{l}\text { El DS es un concepto que alienta a que se } \\
\text { racionalicen los recursos, para no dar tanta } \\
\text { importancia al capital y más a los recursos naturales; } \\
\text { al contrario de lo que se cree no siempre el tener } \\
\text { más, implica estar mejor... }\end{array}$ \\
\hline $\begin{array}{l}\text { Crecimiento } \\
\text { social/económico/político/ambiental } \\
\text { respetando el medio }\end{array}$ & $6 \%$ & $\begin{array}{l}\text { DS es el crecimiento de las sociedades, no solo a } \\
\text { nivel económico sino social, político, etc. respetando } \\
\text { lo que nos rodea, y dejando un ecosistema digno } \\
\text { para las próximas generaciones. }\end{array}$ \\
\hline Controlar lo que consumimos & $6 \%$ & $\begin{array}{l}\text { Controlar lo que consumimos siendo partícipes en la } \\
\text { obtención y eliminación de los recursos. }\end{array}$ \\
\hline Sin clasificar & $6 \%$ & $\begin{array}{l}\text { Disminuir la huella ecológica a través de la EA para } \\
\text { frenar el consumo y alcanzar el DS }\end{array}$ \\
\hline
\end{tabular}

\section{Escribe entre cinco y diez necesidades que tienes en tu propia vida}

En la Tabla VI presentamos un recuento de las respuestas aportadas por los alumnos agrupadas por categorías. El índice medio de respuestas (necesidades) por individuo es de 7,5. Este dígito coincide con la media aritmética del número mínimo y máximo de necesidades que se les solicitaba (entre 5 y 10 ).

\begin{tabular}{|lc|}
\hline \multicolumn{2}{|l|}{$\begin{array}{l}\text { Tabla VI. Categorías identificadas en las respuestas (N=435) de los estudiantes (N=58) a la Cuestión (c) de la } \\
\text { Actividad } 2 \text { ordenadas por porcentaje de las mismas (entre paréntesis se incluyen algunos atributos } \\
\text { significativos asignados a las categorías por los estudiantes) }\end{array}$} \\
\hline CATEGORÍAS DE NECESIDADES & $\begin{array}{l}\text { PORCENTAJE DE } \\
\text { RESPUESTAS }\end{array}$ \\
\hline Alimentación (saludable) & $10,4 \%$ \\
\hline Socialización-Relación (afecto, amigos, ocio, cariño, amor) & $9,5 \%$ \\
\hline $\begin{array}{l}\text { Ámbito afectivo-individual (autoestima, felicidad, independencia, libertad, seguridad, } \\
\text { reconocimiento y posibilidades, superarme a mí mismo, desarrollarme como persona, } \\
\text { intimidad) }\end{array}$ & $7,4 \%$ \\
\hline Transporte (coche propio) & $7,1 \%$ \\
\hline Educación (título B1 de inglés, estudiar más, aprender, acabar la carrera) & $5,5 \%$ \\
\hline Menor contaminación (agua, aire, acústica, limpieza) & $5,3 \%$ \\
\hline Sueño-descanso & $5,1 \%$ \\
\hline Vestir & $5,1 \%$ \\
\hline Otros (aficiones, contenedores para reciclar, uso de electrodomésticos, aprender a conducir) & $5,7 \%$ \\
\hline
\end{tabular}

\section{7. d.1. ¿Has anotado alguna necesidad que esté en conflicto con otra? d.2. ¿Cuál elegirías y cómo tomarías tu decisión?}

En la Tabla VII presentamos un recuento de los porcentajes de respuestas aportadas por los alumnos agrupadas por categorías. Para la primera parte de la cuestión (d.1), el número de respuestas fue de 71 (índice por individuo: 1,22) y para la segunda (d.2) de 43 (índice por individuo: 0,74). 


\begin{tabular}{|c|c|c|c|}
\hline \multicolumn{4}{|c|}{$\begin{array}{l}\text { Tabla VII. Categorías identificadas en las respuestas de los estudiantes }(N=58) \text { a las dos partes de la } \\
\text { Cuestión (d) de la Actividad 2, ordenadas por frecuencia y porcentaje de las mismas }\end{array}$} \\
\hline $\begin{array}{l}\text { CATEGORÍAS DE RESPUESTA } \\
\text { A LA CUESTIÓN d.1 }\end{array}$ & $\begin{array}{l}\text { PORC. DE } \\
\text { RESPUESTAS }\end{array}$ & $\begin{array}{l}\text { CATEGORÍAS DE RESPUESTA } \\
\text { A LA CUESTIÓN d.2 }\end{array}$ & $\begin{array}{l}\text { PORC. DE } \\
\text { RESPUESTAS }\end{array}$ \\
\hline No & $34 \%$ & Las necesidades más básicas & $21 \%$ \\
\hline Viajar/aire limpio & $24 \%$ & $\begin{array}{l}\text { Viajar considerando criterios } \\
\text { ambientales }\end{array}$ & $18 \%$ \\
\hline Alimentación/Origen - otros & $10 \%$ & Aire limpio & $9 \%$ \\
\hline $\begin{array}{l}\text { TIC - Comunicación/Consumo } \\
\text { energético - Contaminación }\end{array}$ & $6 \%$ & Cultivar un huerto propio & $5 \%$ \\
\hline \multirow[t]{2}{*}{ Sin clasificar } & \multirow[t]{2}{*}{$20 \%$} & Alimentación saludable & $5 \%$ \\
\hline & & Ser austero & $5 \%$ \\
\hline
\end{tabular}

\section{8. ¿Qué necesidades deberán satisfacerse primero?}

En la Tabla VIII presentamos un recuento de las respuestas aportadas por los alumnos agrupadas por categorías y ejemplificadas. El número de respuestas fue de 57 (índice por individuo: 0,98).

\begin{tabular}{|c|c|c|}
\hline $\begin{array}{l}\text { CATEGORIAS DE } \\
\text { RESPUESTA }\end{array}$ & $\begin{array}{l}\text { PORCENTAJE } \\
\text { DE RESPUESTAS }\end{array}$ & EJEMPLO DE RESPUESTAS \\
\hline $\begin{array}{l}\text { Las que satisfacen a todos por igual y } \\
\text { al m.a. }\end{array}$ & $21 \%$ & $\begin{array}{l}\text { Debe haber un equilibrio basado en criterios de } \\
\text { equidad y respeto hacia los demás y al medio }\end{array}$ \\
\hline $1^{\circ}$ las primarias, $2^{\circ}$ cuidado del m.a. & $19 \%$ & $\begin{array}{l}\text { Primero se deben de satisfacer las necesidades } \\
\text { primarias y en segundo lugar tener en cuenta las que } \\
\text { puedan perjudicar el m.a. }\end{array}$ \\
\hline Crítica al consumismo & $12 \%$ & $\begin{array}{l}\text { Todo esto es provocado por nuestro afán de } \\
\text { conseguir más y más a cualquier precio, ya que } \\
\text { queremos grandes cantidades de objetos materiales } \\
\text { sin importar el daño que podamos causar a la } \\
\text { naturaleza y las personas }\end{array}$ \\
\hline $\begin{array}{l}1^{\circ} \text { las imprescindibles, } 2^{\circ} \text { las } \\
\text { generadas socialmente }\end{array}$ & $10 \%$ & $\begin{array}{l}\text { Las imprescindibles que tiene todo el mundo para } \\
\text { sobrevivir y } 2^{\circ} \text { las individuales creadas por la } \\
\text { sociedad }\end{array}$ \\
\hline Las de los más necesitados & $9 \%$ & $\begin{array}{l}\text { Las necesidades primarias de las personas que más } \\
\text { lo necesiten }\end{array}$ \\
\hline Prevalencia del poder y el dinero & $7 \%$ & $\begin{array}{l}\text { Gana la necesidad de quien tiene más poder y } \\
\text { dinero, sin importar el bienestar de otras personas y } \\
\text { del m.a. }\end{array}$ \\
\hline Sin clasificar & $10 \%$ & $\begin{array}{l}\text { Las personas intentan cubrir primero sus } \\
\text { necesidades básicas para sobrevivir: alimentación y } \\
\text { vivienda }\end{array}$ \\
\hline
\end{tabular}

\section{9. ¿Qué necesidades sociales, económicas o ambientales agregarías? Indícalas}

En la Tabla IX presentamos los resultados de esta nueva cuestión, agrupados según el apartado de necesidades sociales, económicas y ambientales. Dado que las respuestas mayoritarias se limitaban a enumerarlas no hemos incluido frases a modo de ejemplo. Debemos resaltar que el número de respuestas a las necesidades sociales fue de 148 (índice por individuo: 2,64), 95 para las económicas (índice por individuo: 1,70) y de 119 para las ambientales (índice por individuo: 2,12); a aquellas habría que sumar dos respuestas globales ( $\sin$ distinguir por tipo de necesidad). 
Parece que la formación e intereses de los estudiantes repercutieron en la cantidad de respuestas formuladas.

\begin{tabular}{|c|c|c|}
\hline TIPO DE NECESIDAD & CATEGORÍAS DE RESPUESTA & PORCENTAJE \\
\hline \multirow[t]{6}{*}{ Sociales } & - Aceptación/pertenencia al grupo & $7,4 \%$ \\
\hline & - Asociación/colaboración/cooperación con otras personas & $6,1 \%$ \\
\hline & - Respeto/tolerancia & $6,1 \%$ \\
\hline & - Justicia social & $5,4 \%$ \\
\hline & - Dar y recibir estima/empatía & $5,4 \%$ \\
\hline & - Sin clasificar & $28,6 \%$ \\
\hline \multirow[t]{5}{*}{ Económicas } & - Trabajo remunerado/digno & $19 \%$ \\
\hline & - Obtención de recursos económicos & $9,5 \%$ \\
\hline & - Distribución justa de la riqueza & $7,4 \%$ \\
\hline & - Desarrollo económico & $7,4 \%$ \\
\hline & - Sin clasificar & $26,1 \%$ \\
\hline \multirow[t]{8}{*}{ Ambientales } & - Reducir contaminación & $16,8 \%$ \\
\hline & - Reciclar & $10,9 \%$ \\
\hline & - Respecto y conservación m.a. & $10,9 \%$ \\
\hline & - Utilización de fuentes/recursos renovables & $8,4 \%$ \\
\hline & - Concienciación/formación/información & $7,6 \%$ \\
\hline & - Preservar la biodiversidad & $5,9 \%$ \\
\hline & - Consumir productos ecológicos & $5 \%$ \\
\hline & - Sin clasificar & $16,1 \%$ \\
\hline
\end{tabular}

\section{0. ¿Qué dilemas sobre el DS enfrentas tú todos los días? Relaciónalos}

En la Tabla $X$ presentamos las respuestas a esta última cuestión dadas por los estudiantes $(\mathrm{N}=54)$ agrupadas por categorías e incluyendo un ejemplo de las mismas. El número de respuestas totales fue de 129, incluyendo cuatro respuestas genéricas (índice por individuo: 2,39).

Tabla X. Categorías identificadas en las respuestas de los estudiantes ( $\mathrm{N}=54)$ a la Cuestión (g) de la Actividad 2, porcentajes de las mismas y ejemplos de las respuestas categorizadas (los dilemas se representan conectados por un guión; m.a.: medio ambiente).

\begin{tabular}{|lcl|}
\hline CATEGORIAS & PORCENTAJE & EJEMPLO DE RESPUESTAS \\
\hline $\begin{array}{l}\text { Uso del automóvil-otros } \\
\text { medios de transporte } \\
\text { menos contaminantes }\end{array}$ & $18,6 \%$ & $\begin{array}{l}\text { Otro es que uso el coche en ocasiones cuando también existen } \\
\text { muchos tipos de transporte público, e incluso la bicicleta para } \\
\text { desplazarnos }\end{array}$ \\
\hline $\begin{array}{l}\text { Deseo de reciclar-falta } \\
\text { de contenedores/de } \\
\text { colaboración/por } \\
\text { comodidad }\end{array}$ & $13,2 \%$ & $\begin{array}{l}\text { Un problema que a mi personalmente me preocupa a diario, es } \\
\text { el hecho de querer reciclar la basura y los desperdicios } \\
\text { realizados a lo largo del dia y, pese a haberlos clasificado en } \\
\text { casa, a la hora de bajar la basura solamente encuentras el } \\
\text { contenedor de basura orgánica o los demás contenedores se } \\
\text { encuentran muy lejos del lugar habitual donde hecho la basura }\end{array}$ \\
\hline Ahorro de agua-derroche & & $\begin{array}{l}\text { Uno de los dilemas es que se que hay que ahorrar en el agua } \\
\text { para ducharse pero sin darme cuenta desperdicio más agua de } \\
\text { la necesaria para la ducha, por ello hay que tomar más } \\
\text { conciencia y ahorrar en este tipo de cosas }\end{array}$ \\
& $6,2 \%$ & $\begin{array}{l}\text { El uso de electricidad es otra necesidad que hoy en día se me } \\
\text { ha hecho imprescindible de cubrir, pues la uso tanto para los } \\
\text { electrodomésticos, como para cargar el móvil, como para hacer } \\
\text { que funcionen miles de objetos que necesitan de la corriente }\end{array}$ \\
\hline ahorro de electricidad- & & $\begin{array}{l}\text { Tengo muchos gastos económicos, y esto me dificulta el seguir } \\
\text { estudiando en la Universidad }\end{array}$ \\
\hline Sin clasificar & $5,4 \%$ &
\end{tabular}




\section{Análisis y discusión de los resultados}

A partir de los resultados mostrados en las Tablas I-X, podemos hacer las siguientes inferencias:

- Los estudiantes son conscientes, en general, del grave deterioro ambiental que se traduce en escasez de reciclaje, derroche de recursos, consumismo...

- Las causas son variadas, incluyendo, entre otras: falta de información/formación, escaso compromiso personal y social, débil apoyo institucional, capitalismo...

- Las posibles soluciones pasarían por revertir las causas anteriores, lo que a nivel individual supondría adecuar los comportamientos a la concienciación preexistente, incidiendo para ello en el ámbito formativo desde edades tempranas, en el contexto familiar y con apoyos institucionales eficientes.

- En cuanto a las relaciones EA-DS, se consideran mutuamente dependientes, actuando la EA como subsidiaria estratégica del DS.

- El DS se concibe mayoritariamente como compatibilidad entre cubrir las necesidades básicas humanas y el consumo de los recursos naturales.

- Por lo que respecta a las necesidades básicas de los participantes aparecen las fisiológicas-logísticas, las sociales-afectivas y, en menor medida, las ambientales.

- Las necesidades en conflicto más frecuentes tienen que ver con la alimentación, el transporte y el consumo eléctrico. En cuanto a su elección individual parecen primar criterios ambientales. Cuando se les pregunta a nivel genérico por las necesidades sociales surgen argumentos que tienen que ver con la equidad, la protección ambiental y la crítica a la sociedad del consumo. Si se les da la opción de agrupar las necesidades en sociales, económicas y ambientales, las primeras prevalecen, seguidas por las ambientales y las económicas, emergiendo un destacado número de valores.

- Por último, los dilemas personales que tienen que ver con el DS se centran especialmente en el uso de los medios de transporte, el reciclaje y el consumo de agua y electricidad. Ello constituye sin duda un necesario punto de partida para facilitarles herramientas de actuación.

El presente trabajo incide en una de las dimensiones de la EA que más ha evolucionado en las últimas décadas hasta el punto de poder caracterizarla con el término socioambiental. Con ello se pretende reconocer que los problemas ambientales son ante todo problemas sociales y que su resolución pasa ineludiblemente por la participación y transformación sociales (Iglesias y Meira, 2007).

En nuestro caso hemos querido poner de manifiesto esa íntima relación a través de una experiencia curricular singular: cómo estudiantes del grado de ES perciben los grandes dilemas a los que se enfrenta la EA, en general, y el DS en particular.

Las actividades planteadas a dichos estudiantes incitan a la reflexión y toma de postura ante los referidos dilemas, haciéndolo al inicio del curso y posteriormente cuando ya han sido introducidos en los conceptos básicos de la EA. Su análisis conjunto nos ha permitido obtener una radiografía de sus conocimientos, creencias, valores y expectativas socioambientales que reproducen con riqueza y diversidad las inquietudes de este colectivo de jóvenes, desechando la idea de que son meros espectadores pasivos de lo que a su alrededor ocurre. Asimismo avala la necesidad de ambientalizar curricularmente los estudios del grado de ES, tal y como otros autores ya han reclamado (De la Osa y Azara, 2012; Amador y Esteban, 2011). 


\section{Referencias}

Alonso, B. (2010). Historia de la Educación Ambiental. La Educación ambiental en el siglo XX. Asociación Española de Educación Ambiental. Recuperado de http://ae-ea.es/wpcontent/uploads/2016/06/Historia-de-la-educacionambiental.pdf

Amador, LV. y Esteban, M. (2011). Desde la educación social a la educación ambiental. Hacia una intervención educativa socioambiental. Revista de Humanidades, 18, 147-160.

Aramburuzabala, P., Cerrillo, R. y Tello, I. (2015). Aprendizajeservicio: una propuesta metodológica para la introducción de la sostenibilidad curricular en la universidad. Profesorado, 19(1), 78-95.

CADEP-CRUE (2012). Directrices para la introducción de la sostenibilidad en el curriculum. Actualización de la declaración institucional aprobada en 2005. http://www.crue.org/Sostenibilidad/CADEP/Paginas/Documen tos.aspx?Mobile $=0$

Comisión Brundtland (1987). Nuestro Futuro Común. Oxford: Oxford University Press.

Conroy, C., \& Litvinoff, M. (1988). The Greening of Aid. London: Earthscan.

Ekins, P. (ed.) (1986). The Living Economy. London: Routledge.

Feinstein, NW., \& Kirchgasler, K. (2015). Sustainability in Science Education? How the next generation science standards approach Sustainability, and Why it matters. Science Education, 99 (1), 121-144.

García, JE. (2004). Educación Ambiental, constructivismo y complejidad. Sevilla: Ed. Diada.

Geli, AM. (2002). Introducción. Universidad, Sostenibilidad y Ambientalización Curricular. En M.G. Junyent, A.M. Geli y E. Arbat (Eds.), Ambientalización curricular de los estudios superiores. (pp. 11-18). Girona: Universidad de Girona, Servicio de Publicaciones.

Hansmann, R. (2009). Linking the Components of a University Program to the Qualification Profile of Graduates: The Case of a Sustainability-Oriented Environmental Science Curriculum. Journal of Research in Science Teaching, 46(5), 537-569.

Iglesias, L. y Meira, P. (2007). De la Educación Ambiental a la Educación Social o viceversa. Educación Social, 35, 13-27.

Jiménez-Fontana, R., García-González, E., Azcárate, P. y Navarrete, A. (2015). Dimensión ética de la sostenibilidad curricular en el sistema de evaluación de las aulas universitarias. El caso de la enseñanza aprendizaje de las Ciencias. Revista Eureka sobre Enseñanza y Divulgación de las Ciencias 12(3), 536-549.

Lucas, A. (1972). Environment and Environmental Education: Conceptual issues and Curriculum Implications. PhD Dissertation Ohio State University.

Luque, A.I. y Perales, F.J. (2016). La "ambientalización" de los estudios de ciencias ambientales en España. Didáctica de las Ciencias Experimentales y Sociales, 30(1), 151-169.
Meadows, DH., Meadows, DL., Randers, J., \& Behrens, WW. (1972). The Limits to Growth: a report for the Club of Rome's project on the predicament of mankind. New York: Universe Books.

Medir, R.M., Heras, R. y Magin, C. (2016). Una propuesta evaluativa para actividades de educación ambiental para la sostenibilidad. Educación XX1, 19(1), 331-355.

Osa, de la J.A. y Azara, M.P. (2012). La educación ambiental es (también) educación social. Comunicación al VI Congreso Estatal de Educación Social. Valencia 3-5 mayo. http://www.aragon.es

Perales, FJ. y Gutiérrez, J. (2010). Claves de cooperación interdisciplinar: una visión retrospectiva de la Educación Ambiental desde su trayectoria en la Universidad de Granada. Revista Eureka sobre Enseñanza y Divulgación de las Ciencias, 7, 400-412.

Sauvé, L. (2004). Una cartografía de Corrientes en Educación Ambiental. Cátedra de Investigación de Canadá en Educación Ambiental. Universidad de Québec. En: Sato M. Carvalho I. (orgs.) La investigación en Educación Ambiental. Montreal, Canadá

Stauffacher, M., Walter, A.I., Lang, D.J., Wiek, A., \& Scholz, R.W. (2006). Learning to research environmental problems from a functional socio-cultural constructivism perspective. The transdisciplinary case study approach. International Journal of Sustainability in Higher Education, 7(3), 252-275.

Tilbury, D. (2011). Education for Sustainable Development. An Expert Review of Processes and Learning. UNESCO: Paris.

Tudela, F. (1990). Desarrollo y Medio Ambiente en América Latina y el Caribe. Una visión evolutiva. Madrid: Ministerio de Obras Públicas y Urbanismo.

Vilches, A. y Gil, D. (2015). Ciencia de la Sostenibilidad: ¿Una nueva disciplina 0 un nuevo enfoque para todas las disciplinas? Revista Iberoamericana de Educación, 69(1), 3960. 